\title{
Lidil
}

Revue de linguistique et de didactique des langues

56 | 2017

Grammaires et littéracies

\section{Les connecteurs, de la grammaire à la littéracie}

Connectors, from Grammar to Literacy

\section{Sabina Mahmudova}

\section{OpenEdition}

\section{Journals}

Édition électronique

URL : http://journals.openedition.org/lidil/4757

DOI : $10.4000 /$ lidil.4757

ISSN : 1960-6052

\section{Éditeur}

UGA Éditions/Université Grenoble Alpes

\section{Édition imprimée}

ISBN : 978-2-37747-024-2

ISSN : $1146-6480$

Référence électronique

Sabina Mahmudova, "Les connecteurs, de la grammaire à la littéracie », Lidil [En ligne], 56 | 2017, mis en ligne le 01 novembre 2017, consulté le 19 avril 2019. URL : http://journals.openedition.org/ lidil/4757 ; DOI : 10.4000/lidil.4757

Ce document a été généré automatiquement le 19 avril 2019

(C) Lidil 


\title{
Les connecteurs, de la grammaire à la littéracie
}

\author{
Connectors, from Grammar to Literacy
}

Sabina Mahmudova

\section{Introduction}

1 La littéracie est au cœur de la réflexion sur l'apprentissage de la lecture-écriture. Le terme désigne :

l'ensemble des activités humaines qui impliquent l'usage de l'écriture, en réception et en production. Elle met un ensemble de compétences de base, linguistiques et graphiques, au service de pratiques, qu'elles soient techniques, cognitives, sociales ou culturelles. (Barré-De Miniac, Brissaud \& Rispail, 2004, p. 11)

Elle s'applique aussi bien à l'apprentissage de la langue maternelle dans sa dimension écrite qu'à celui d'une langue étrangère, associant des savoirs et des savoir-faire parmi lesquels figure la grammaire dans ses multiples facettes (grammaire de phrase et de texte). Dans la perspective de la didactique des langues, écrire, c'est produire du sens dans un but communicationnel. Le CECRL (Cadre européen commun de référence pour les langues) définit différentes « compétences communicatives langagières » (CECRL, p. 86) de «l'utilisateur/apprenant». Parmi celles-ci, la compétence pragmatique traite de la connaissance que l'utilisateur/l'apprenant a des principes selon lesquels les messages sont :

- organisés, structurés et adaptés,

- utilisés pour la réalisation de fonctions communicatives,

- segmentés selon des schémas interactionnels et transactionnels. (CECRL, p. 96)

Les conditions requises sont d'avoir quelque chose à dire, savoir à qui adresser son message et pouvoir écrire en tenant compte du type de texte à produire. Lors de l'apprentissage d'une langue étrangère, l'apprenant commence par de brefs énoncés. Aux niveaux supérieurs, le développement d'une compétence discursive, dont les 
composantes sont inventoriées dans cette section, devient de plus en plus important (CECRL, p. 96).

On s'intéresse dans cette étude à l'utilisation des connecteurs par des étudiants azerbaïdjanais. Après avoir situé les connecteurs dans la compétence discursive et les avoir définis pour comprendre leur rôle dans la production écrite, nous décrirons et expliquerons les usages des apprenants à partir de l'analyse de leurs productions. Ensuite, à partir de cette observation, nous présenterons des moyens possibles pour améliorer la maitrise des connecteurs.

\section{La compétence discursive}

Nous ne produisons jamais un texte en un seul jet. L'écrit oblige à faire des retours sur la production écrite, des corrections et des révisions, des ajouts et des suppressions. L'écrit suit également des objectifs divers, comme présenter des informations basées sur les connaissances acquises, exprimer des sentiments, transmettre ses impressions. L'expression écrite est par conséquent libre, créative et peut engager le locuteur de façon personnelle, mais pas toujours (écrits administratifs, comptes rendus...).

6 La rédaction d'un texte signifie la production d'énoncés cohérents, structurés, sans oublier bien évidemment la progression thématique. La compétence d'écrire exige la mise en œuvre d'habiletés et de stratégies que l'apprenant acquiert graduellement. Il faut avant tout comprendre que le texte écrit n'est pas une simple succession de phrases, mais une unité complexe. À part le respect des règles grammaticales, la maitrise du vocabulaire, il faut savoir surveiller la structuration du texte, qui, comme celle de la phrase, obéit à des règles plus ou moins strictes (Cornaire \& Raymond, 1999). Cette entreprise devient encore plus compliquée quand il s'agit d'écrire dans une langue étrangère, en l'occurrence en français, qui possède une structure différente de celle de l'azerbaidjanais.

7 Notre choix des connecteurs s'appuie principalement sur le fait que les apprenants sont souvent invités à produire des textes en français, sur des sujets variés («Est-il possible de réussir sans trahir personne ?»; "L'ordinateur vous isole du monde. Qu'en pensezvous ?»; «Il faudrait interdire toute sorte de censure. Oui ou non ? Dites pourquoi. »). Les connecteurs logiques, qui font partie de la compétence pragmatique, jouent un rôle clé dans la production d'écrits. Leur emploi est en bonne partie déterminé par le type de texte (Adam, 2011). Un texte argumentatif a besoin de connecteurs pour que sa structuration soit clairement perceptible (Adam, 2017). Et même si les connecteurs sont utilisés dans le discours oral, c'est dans le discours écrit qu'ils sont indispensables, puisque celui-ci est dépourvu des marques paralinguistiques de l'oral pour marquer sa structuration. Et à l'écrit, la variété du choix des connecteurs permet d'exprimer des nuances parfois subtiles (ex. des connecteurs d'opposition: mais, cependant, néanmoins, pourtant, toutefois, etc.) (Ruquet \& Quoy-Bodin, 1998).

Dans l'enseignement de la langue française, nous rencontrons, en tant qu'enseignante, une série de lacunes dans les productions écrites. Quel que soit le niveau de l'apprenant le problème le plus saillant est celui de la liaison des idées et des phrases dans un texte. Avoir des idées (claires souvent) sur le sujet n'aide pas pour autant l'apprenant à présenter une production écrite cohérente. Nous ne pouvons pas ne pas mentionner l'importance de la production écrite lors des examens de TCF (test de connaissance du 
français) et de DELF/DALF (diplômes en langue française). Ces tests, qui définissent le niveau de l'apprenant en français, accordent beaucoup d'attention à la cohérence et la cohésion. Pourtant, bien que les apprenants reconnaissent l'importance des connecteurs, ils hésitent à les utiliser. Nous trouvons sur les copies des examens des idées juxtaposées, des phrases isolées. Les textes sont compréhensibles, certes, et conformes à la logique, mais l'absence des transitions entre les phrases et les étapes du raisonnement affaiblit la cohérence et la cohésion textuelles. Par conséquent, les résultats lors des épreuves d'expressions écrites sont moins satisfaisants que les résultats des autres compétences comme la structure de la langue, la compréhension orale, etc.

9 Nous étudierons comment les apprenants utilisent les connecteurs dans leurs productions écrites et par quels autres moyens ils marquent les relations entre les phrases. Puis nous verrons comment aider les apprenants à développer et diversifier l'emploi des connecteurs là où ils sont nécessaires.

\section{Qu'est-ce qu'un connecteur?}

Différents éléments contribuent à la cohésion du texte, notamment les anaphores qui tissent des fils conducteurs en reliant des expressions référentielles, et les connecteurs qui assurent le chainage logique et argumentatif du texte.

Dans l'enchainement linéaire du texte, les connecteurs sont des termes de liaison et de structuration: ils contribuent à la structuration du texte et du discours en marquant des relations entre les propositions ou entre les séquences qui composent le texte et en indiquant les articulations du discours. (Riegel, Pellat \& Rioul, 2016, p. 1044)

11 Les connecteurs explicitent les relations, notamment logiques, à l'intérieur du texte et aident à faire percevoir son organisation par le lecteur.

On établit deux listes de connecteurs, l'une restreinte, l'autre ouverte. Au sens restreint, les connecteurs sont les termes qui assurent la liaison à l'intérieur d'une phrase complexe. On y inclut les conjonctions de coordination (mais, ou, et, donc, or, ni, car) et les conjonctions de subordination (pour que, de peur que, bien que, tandis que, etc.) qui sont des mots-outils invariables qui servent à introduire une proposition subordonnée. Au sens large, les connecteurs sont tous les termes qui assurent l'organisation d'un texte. Avec les conjonctions (de coordination et de subordination), on emploie également :

- les adverbes de liaison (ainsi, alors, certes, néanmoins, cependant, etc.). Ces adverbes servent à relier deux phrases et notamment à effectuer un balisage de la progression textuelle, soulignent l'organisation générale du discours et facilitent l'orientation du lecteur dans sa progression;

- les groupes prépositionnels. Par son sens, la préposition permet d'exprimer différents rapports logiques entre les éléments qu'elle lie, par exemple le temps (avant, depuis, en), le lieu (dans, chez, sur), le but (pour, afin de), l'opposition (contre, malgré), etc.

\section{Considérations méthodologiques}

13 Pour analyser de plus près l'utilisation des connecteurs et des autres moyens de liaison dans la production écrite, nous avons opté pour une méthode expérimentale de manière à faire un état des lieux. Dans quelle mesure nos apprenants utilisent-ils des connecteurs? 
Quelles stratégies d'écriture adoptent-ils pour conserver la cohérence en marquant les relations entre les phrases par d'autres moyens?

Pour cette recherche exploratoire, nous avons fait une étude sur les textes produits par neuf apprenants du niveau avancé, qui se situe entre B2 et C1 du Cadre européen commun de référence pour les langues. Les apprenants dont les écrits sont analysés sont des étudiants des facultés de la traduction et de philologie de l'Université des langues d'Azerbaïdjan. Nous leur avons demandé de rédiger chacun trois textes à dominante argumentative, à partir de trois sujets qui sont de même niveau que ceux que l'on trouve dans les épreuves du TCF et du DELF/DALF. Ces sujets sont les suivants :

Sujet 1 (S1). Certaines personnes aiment rester seules: d'autres au contraire, aiment avoir de la compagnie (des amis-es). Et vous, que préférez-vous? Rédigez un texte dans lequel vous développerez votre avis à l'aide d'arguments précis. ( \pm 300 mots)

Sujet 2 (S2). Écrivez une critique d'un film ou d'un roman que vous avez vu ou lu. Précisez les points positifs et négatifs. ( \pm 300 mots $)$

Sujet 3 (S3). Voici quelques lignes de l'éditorial du magazine Femme Actuelle (17-23 mars 2008) dont l'objectif est :

a) d'informer sur la situation de l'eau courante en Afrique ;

b) de sensibiliser le lecteur à la manière dont, avec des gestes simples, il peut contribuer à une meilleure consommation de l'eau dans son quotidien.

En respectant les deux fonctions du texte ainsi que le style choisi par l'éditorialiste, continuez la rédaction de l'éditorial. ( \pm 150 mots)

Pour quelques gouttes d'eau

Muriel Picard, rédactrice en chef

Ouvrir le robinet et voir couler de l'eau propre à la consommation n'a pour nous rien d'extraordinaire. Mais, pour des millions de gens, cela tient encore du miracle. Une simple fontaine change même radicalement la vie dans ces villages d'Afrique où les femmes et les fillettes doivent aller chercher l'eau à des kilomètres. Échapper à cette corvée, c'est plus de temps pour aller à l'école, cultiver un potager, mettre en route des activités qui feront vivre toute une famille...

Nous avons pu analyser 9 productions pour le premier sujet, 9 productions pour le deuxième sujet et 5 productions pour le troisième sujet. Dix jours ont été accordés aux apprenants pour cette production écrite à la maison. Nous souhaitions qu'ils aient un temps suffisant pour la rédaction, pour pouvoir bien réfléchir à la structuration de leur texte, trouver les idées et faire une présentation convenable. Comme le public choisi avait le niveau avancé, nous n'avons pas eu besoin de leur donner des explications complémentaires sur les sujets. Nous n'avons pas révélé l'objet d'étude de cette expérimentation pour voir comment nos étudiants emploient spontanément les connecteurs dans leurs productions.

L'analyse des textes est d'ordre descriptif. En examinant les copies des étudiants, nous avons établi un mode de classement qui distingue l'emploi, l'absence et le remplacement des connecteurs, au niveau de la phrase et au niveau du texte dans son ensemble. Ce classement permet de repérer la variété des connecteurs employés, de distinguer leurs emplois et de révéler des moyens alternatifs employés. 


\section{Résultats des observations et interprétation des données}

\subsection{Tableaux récapitulatifs}

Les tableaux quantitatifs ci-dessous résument les résultats des analyses.

Tableau 1. - Emploi des connecteurs dans les rédactions du sujet 1.

\begin{tabular}{|c|c|c|c|c|c|c|c|}
\hline \multirow[b]{3}{*}{ Apprenant } & \multicolumn{7}{|c|}{ CONNECTEURS } \\
\hline & \multicolumn{3}{|c|}{$\begin{array}{c}\text { Entre phrases ou propositions } \\
\text { indépendantes }\end{array}$} & \multirow[b]{2}{*}{$\begin{array}{c}\text { Absence ou } \\
\text { substitution } \\
\text { des } \\
\text { connecteurs }\end{array}$} & \multicolumn{3}{|c|}{$\begin{array}{l}\text { Entre propositions } \\
\text { subordonnées }\end{array}$} \\
\hline & $\begin{array}{c}\text { Conj. de } \\
\text { coordination }\end{array}$ & $\begin{array}{c}\text { Adverbe } \\
\text { de } \\
\text { liaison }\end{array}$ & $\begin{array}{c}\text { Groupe } \\
\text { prépositionnel }\end{array}$ & & $\begin{array}{c}\text { Conj. de } \\
\text { subordination }\end{array}$ & $\begin{array}{c}\text { Gr. } \\
\text { prép. } \\
\text { et } \\
\text { adv. } \\
\text { de } \\
\text { liaison }\end{array}$ & Autres \\
\hline A1 & 1 & 1 & 2 & 4 & 1 & 0 & 0 \\
\hline A2 & 1 & 1 & 1 & 5 & 2 & 0 & 1 \\
\hline A3 & 3 & 0 & 0 & 4 & 3 & 0 & 1 \\
\hline A4 & 1 & 1 & 2 & 0 & 2 & 0 & 0 \\
\hline A5 & 0 & 1 & 3 & 4 & 2 & 0 & 0 \\
\hline A6 & 4 & 1 & 1 & 2 & 4 & 1 & 1 \\
\hline A7 & 1 & 4 & 0 & 2 & 5 & 1 & 1 \\
\hline A8 & 7 & 1 & 1 & 2 & 1 & 2 & 0 \\
\hline A9 & 4 & 2 & 0 & 2 & 0 & 0 & 0 \\
\hline & 22 & 12 & 10 & 25 & 20 & 4 & 4 \\
\hline
\end{tabular}

Le premier sujet est une argumentation qui incite à faire un choix entre deux attitudes morales et sociales. La consigne ne demande pas explicitement l'emploi des connecteurs. En revanche, elle invite à articuler plusieurs arguments. Les étudiants privilégient les connecteurs qu'ils ont appris à maitriser dans le cadre de la grammaire de phrase, d'où le nombre important des conjonctions de coordination (22) et de subordination (20). Ils ont aussi recours à d'autres procédés, comme éviter les connecteurs ou y suppléer (25). 
Tableau 2. - Emploi des connecteurs dans les rédactions du sujet 2.

\begin{tabular}{|c|c|c|c|c|c|c|c|}
\hline \multirow[b]{3}{*}{ Apprenant } & \multicolumn{7}{|c|}{ CONNECTEURS } \\
\hline & \multicolumn{3}{|c|}{$\begin{array}{c}\text { Entre phrases ou propositions } \\
\text { indépendantes }\end{array}$} & \multirow[b]{2}{*}{$\begin{array}{l}\text { Absence ou } \\
\text { substitution } \\
\text { des } \\
\text { connecteurs }\end{array}$} & \multicolumn{3}{|c|}{$\begin{array}{l}\text { Entre propositions } \\
\text { subordonnées }\end{array}$} \\
\hline & $\begin{array}{l}\text { Conj. de } \\
\text { coordination }\end{array}$ & $\begin{array}{c}\text { Adverbe } \\
\text { de } \\
\text { liaison }\end{array}$ & $\begin{array}{c}\text { Groupe } \\
\text { prépositionnel }\end{array}$ & & $\begin{array}{c}\text { Conj. de } \\
\text { subordination }\end{array}$ & $\begin{array}{c}\text { Gr. } \\
\text { prép. } \\
\text { et } \\
\text { adv. } \\
\text { de } \\
\text { liaison }\end{array}$ & Autres \\
\hline A1 & 4 & 0 & 1 & 1 & 2 & 0 & 0 \\
\hline A2 & 2 & 0 & 0 & 0 & 1 & 0 & 1 \\
\hline A3 & 1 & 0 & 0 & 2 & 2 & 0 & 0 \\
\hline A4 & 0 & 0 & 0 & 0 & 0 & 0 & 0 \\
\hline A5 & 0 & 2 & 0 & 0 & 0 & 0 & 0 \\
\hline A6 & 4 & 0 & 0 & 0 & 0 & 0 & 0 \\
\hline A7 & 2 & 4 & 1 & 2 & 6 & 2 & 0 \\
\hline A8 & 4 & 2 & 3 & 1 & 0 & 0 & 0 \\
\hline \multirow[t]{2}{*}{ A9 } & 1 & 2 & 0 & 0 & 1 & 0 & 0 \\
\hline & 18 & 10 & 5 & 6 & 12 & 2 & 1 \\
\hline
\end{tabular}

19 À la différence du premier sujet, le deuxième demande une composition organisée («Précisez les points positifs et négatifs») et est moins exigeant dans la diversification des valeurs logiques. Ainsi, les scripteurs n'ont pas eu besoin d'utiliser une aussi grande variété de connecteurs logiques. Ce qui nous confirme que l'emploi des connecteurs logiques est déterminé par le sujet lui-même. Les étudiants ont eu tendance à utiliser les conjonctions de coordination pour organiser leur texte. Par exemple A6 n'a employé que 4 conjonctions de coordination et aucun autre connecteur pour construire son texte. Et globalement, ils n'ont pas eu besoin d'écrire des phrases complexes parce qu'ils peuvent se contenter d'une narration, ce qui n'était pas le cas dans le premier sujet.

Tableau 3. - Emploi des connecteurs dans les rédactions du sujet 3.

\begin{tabular}{|l|c|c|}
\hline \multirow{2}{*}{} & \multicolumn{2}{|c|}{ C O N N E C T E U R S } \\
\cline { 2 - 3 } & $\begin{array}{c}\text { Entre phrases ou propositions } \\
\text { indépendantes }\end{array}$ & $\begin{array}{c}\text { Entre propositions } \\
\text { subordonnées }\end{array}$ \\
\cline { 2 - 3 } Apprenant & $\begin{array}{c}\text { Absences ou } \\
\text { substitution } \\
\text { Lidil, } 56 \mid 2017\end{array}$ & $\begin{array}{c}\text { des } \\
\text { connecteurs }\end{array}$ \\
\end{tabular}




\begin{tabular}{|c|c|c|c|c|c|c|c|}
\hline & $\begin{array}{l}\text { Conj. de } \\
\text { coordination }\end{array}$ & $\begin{array}{c}\text { Adverbe } \\
\text { de } \\
\text { liaison }\end{array}$ & $\begin{array}{l}\text { Groupe } \\
\text { prépositionnel }\end{array}$ & & $\begin{array}{c}\text { Conj. de } \\
\text { subordination }\end{array}$ & $\begin{array}{c}\text { Gr. } \\
\text { prép. } \\
\text { et } \\
\text { adv. } \\
\text { de } \\
\text { liaison }\end{array}$ & Autres \\
\hline A1 & 1 & 1 & 0 & 0 & 2 & 0 & 0 \\
\hline A2 & 2 & 0 & 0 & 0 & 0 & 0 & 0 \\
\hline A6 & 2 & 2 & 0 & 2 & 0 & 0 & 0 \\
\hline A7 & 2 & 2 & 2 & 1 & 0 & 0 & 0 \\
\hline A9 & 4 & 1 & 1 & 1 & 1 & 0 & 0 \\
\hline & 11 & 6 & 3 & 4 & 3 & 0 & 0 \\
\hline
\end{tabular}

Le besoin en connecteurs est moins important puisqu'il s'agit de poursuivre un paragraphe avec un volume moindre. Comme la consigne invite à continuer une idée déjà présente, un texte déjà amorcé, les apprenants n'ont pas eu besoin d'utiliser un grand nombre de connecteurs pour relier les phrases ni de trouver des équivalents.

Tableau 4. - Récapitulatif.

\begin{tabular}{|c|c|c|c|c|c|c|c|}
\hline \multirow[b]{3}{*}{ Apprenant } & \multicolumn{7}{|c|}{ C ONNECTEURS } \\
\hline & \multicolumn{3}{|c|}{$\begin{array}{c}\text { Entre phrases ou propositions } \\
\text { indépendantes }\end{array}$} & \multirow[b]{2}{*}{$\begin{array}{c}\text { Absence ou } \\
\text { substitution } \\
\text { des } \\
\text { connecteurs }\end{array}$} & \multicolumn{3}{|c|}{$\begin{array}{l}\text { Entre propositions } \\
\text { subordonnées }\end{array}$} \\
\hline & $\begin{array}{l}\text { Conj. de } \\
\text { coordination }\end{array}$ & $\begin{array}{c}\text { Adverbe } \\
\text { de } \\
\text { liaison }\end{array}$ & $\begin{array}{c}\text { Groupe } \\
\text { prépositionnel }\end{array}$ & & $\begin{array}{c}\text { Conj. de } \\
\text { subordination }\end{array}$ & $\begin{array}{c}\text { Gr. } \\
\text { prép. } \\
\text { et } \\
\text { adv. } \\
\text { de } \\
\text { liaison }\end{array}$ & Autres \\
\hline A1 & 6 & 2 & 3 & 5 & 5 & 0 & 0 \\
\hline A2 & 5 & 1 & 1 & 5 & 3 & 0 & 2 \\
\hline A3 & 4 & 0 & 0 & 6 & 5 & 0 & 1 \\
\hline A4 & 1 & 1 & 2 & 0 & 2 & 0 & 0 \\
\hline A5 & 0 & 3 & 3 & 4 & 2 & 0 & 0 \\
\hline A6 & 10 & 3 & 1 & 4 & 4 & 1 & 1 \\
\hline A7 & 5 & 10 & 3 & 5 & 11 & 3 & 1 \\
\hline
\end{tabular}




\begin{tabular}{|c|c|c|c|c|c|c|c|}
\hline A8 & 11 & 3 & 4 & 3 & 1 & 2 & 0 \\
\hline A9 & 9 & 5 & 1 & 3 & 2 & 0 & 0 \\
\hline & 51 & $\mathbf{2 8}$ & $\mathbf{1 8}$ & 35 & 35 & $\mathbf{6}$ & $\mathbf{5}$ \\
\hline
\end{tabular}

\subsection{Un usage déséquilibré des connecteurs}

Dans le cadre de l'enquête, les apprenants sont plus à l'aise avec les conjonctions de coordination, utilisent moins les adverbes de liaison ( 28 entre les phrases) et encore moins les groupes prépositionnels (18 entre les phrases).

Malgré leur usage récurrent, nous trouvons les connecteurs plus souvent entre les éléments de la même phrase (intraphrastiques) (97 pour les 3 sujets) et moins entre les phrases du texte (interphrastiques) (46 pour les 3 sujets) : la compétence discursive des apprenants est moins assurée que leur compétence grammaticale qui se limite à la grammaire de phrase.

\subsubsection{Emplois des conjonctions de coordination}

La conjonction de coordination et est utilisée d'une manière « polysémique » en début de phrase ou de proposition. Les apprenants lui attribuent plusieurs valeurs sémantiques - hors l'addition - ; les exemples qui suivent montrent la variété des sens de et :

A1 S1 - [...] J'aspire toujours après une longue journée de travail ou la soirée bruyante. Je le préfère souvent au sorties et invitations. Je préfère même voyager seule ce que je fais assez souvent. Et rien n'est mieux pour moi que le temps passé avec un bon livre ou un film ou même sans rien faire du tout, sans personne ${ }^{1}$.

La conjonction et utilisée en début de la phrase sert à ajouter un argument complémentaire plus fort (« et rien n'est mieux »).

A1 S2 - Ce que j'aime le plus dans la vie c'est voyager, lire et regarder les films anciens, ainsi que les séries. Et puisqu'on ne peut pas raconter pleinement de livre en respectant le limite de 300 mots, je vais écrire à propos d'une série que je viens de terminer à regarder.

A9 S1 - [...] je préfère souvent rester tout seul avec une ou deux personnes maximum. Et je dois préciser que ces personnes sont ceux que j'ai choisis à l'issue de certains temps.

Le et ajoute dans ce cas une caractéristique, pour préciser l'idée exprimée.

A8 S1 - A ce moment-là on a envie d'être en solitude et ce n'est pas mal.

A8 S1 - Donc à mon avis la solitude a plusieurs côtés curieux et, en effet, rester seul n'est pas si affreux.

Dans les deux derniers exemples, et introduit un développement explicatif.

La conjonction mais a elle aussi plusieurs sens différents dans les essais des apprenants.

A6 S2 - Je ne peux pas dire que c'est sa meilleure œuvre, mais le livre m'a parut intéressant et passionnant ce qu'est démontré par le fait que je l'ai fini en cinq jours.

Mais exprime dans cet exemple la concession et introduit la mention d'un intérêt personnel qui s'oppose à l'évaluation générale.

A9 S3 - Il y a en certains d'autre qui peuvent se manifester il n'ont pas de moyens financiers pour venir au secours, donc il peuvent rien faire. Mais c'est bien là qu'il peuvent se tromper. 
La conjonction mais joue bien le rôle d'une opposition entre les opinions dans cet exemple : le scripteur s'oppose à une opinion commune pour avancer une idée centrale dans son essai.

A8 S1 - S'éloigner du milieu habituel est assez difficile pour nous. Mais parfois on en a marre de cette société.

Mais associé à parfois introduit ici un argument contraire qui contredit celui de la phrase précédente.

\subsubsection{Emplois des conjonctions de subordination}

L'emploi des conjonctions de subordination est également intéressant. Les conjonctions de subordination sont fréquemment utilisées; nous en avons repéré beaucoup pour la concession, la cause et l'opposition et très peu (voire aucune) pour la conséquence.

A1 S1 - Les moments pareils m'aident à rétablir l'énergie que je perds rapidement vu que mon travail est entièrement lié à la communication avec les gens de toutes sortes.

A1 S3 - Cela n'exige aucun effort, et même si c'était le cas il faut faire tout ce qui est possible pour préserver ce que nous reste dans une telle petite quantité.

A3 S1 - Je pense surtout que avec l'âge on ressent davantage cette envie d'avoir quelqu'un à côté. Les gens sont tous différents et chacun lorsqu'il cherche quelqu'un il a des critères de choix et des objectifs à réaliser grâce à cette rencontre.

A7 S2 - C'est un film grandement humaniste, et il faut voir, revoir, l'avoir toujours dans la tête, et montrer dans toutes les écoles, les universités, pour que tout le monde sache que la vie des suffragettes n'était pas du tout facile.

A8 S1 - Parfois cela permet de trouver contre toute attente la solution extraordinaire d'un vieux problème. Tandis qu'avant on ne pouvait pas le résoudre.

\subsubsection{Autres connecteurs logiques}

Les autres connecteurs logiques comme les adverbes de liaison et les locutions prépositives ne sont pas aussi fréquemment utilisés que l'on s'y serait attendu.

A9 S1 - Néanmoins, un peu de solitude n'a encore dérangé personne.

A8 S2 - Alors, au cours de tous les saisons les familles nobles mènent la lutte pleine de violences, d'intrigues, d'assassinats, de trahisons pour le trône en ne comprenant pas que la vraie guerre est encore à l'avenir.

A2 S2 - Malgré le mauvais temps Grace monte Pilgrim un jour de janvier froid et ce qui devait arriver arrive.

\subsubsection{Absence de connecteurs}

Les absences des connecteurs sont fréquentes dans les productions écrites. Les scripteurs se contentent souvent d'une simple juxtaposition des phrases et évitent l'emploi des connecteurs. Cela s'expliquerait-il par l'hésitation ou par la non-maitrise du connecteur?

A1 S1 - Peu de gens aiment être seul. La solitude les déprime, il leur faut toujours quelqu'un près d'eux, ils ne supportent pas le silence qui les entoure, ils ne peuvent pathologiquement pas passer le temps sans les autres.

\subsubsection{Les cas de liaison mixte}

Dans le cas des écrits que nous avons analysés, il s'agit d'une stratégie d'écriture que les étudiants ont adoptée : au lieu d'employer seulement des connecteurs, ils font appel à des 
outils qui associent la connexité et l'anaphore. Par cette stratégie, ils marquent qu'ils sont soucieux d'indiquer la structuration de leurs textes.

A2 S1 - Les meilleurs œuvres d'art ont été créés grâce à la solitude et incompréhension. Les grands esprits de l'humanité étaient solitaires. En dépit de cela chaque grand maître a eu sa muse, de laquelle il s'inspirait. Cela veut dire que même si tu es seule physiquement, tu dois avoir un pouvoir spirituel.

Le GPrep équivaut à un connecteur d'opposition (pourtant, néanmoins, etc.) ; cela est anaphorique : il reprend globalement le contenu des phrases précédentes.

A1 S3 - Le manque d'eau entraîne la perte de récolte et cela peut résulter en une grande famine très bientôt.

L'apprenant exprime l'idée de conséquence par le verbe résulter et charge sa conjonction de coordination et d'une valeur de conséquence.

A2 S1 - J'aime être avec ma famille et mes amis, et je trouve qu'ils me soutiennent dans tout ce que je fais. C'est dans le cas où on manque de compréhension de la part de nos proches qu'on ressent l'envie de rester seul. Etre seul est nuisible pour la santé.

"C'est dans le cas où » sert de liaison entre les phrases. "C'est dans le cas où [...] qu'on ressent » exprime un lien de cause à conséquence.

A6 S1 - Pour être heureux il fait avoir un soutien, mais les gens solitaires n'en ont pas. D'ici proviennent la dépression et l'ignorance.

D'ici impropre (il faudrait de là) est aussi une suppléance par la voie anaphorique exprimant la conséquence.

A2 S1 - Les gens sont tous différents et chacun lorsqu'il cherche quelqu'un il a des critères de choix et des objectifs à réaliser grâce à cette rencontre. Les uns en décèlent la possibilité de s'épanouir, les autres cherchent justement quelqu'un qui serait capable d'écouter les problèmes d'autrui.

L'association des indéfinis les uns, les autres, est utilisée pour exprimer la division du tout en ses parties.

A1 S1 - Une hypothèse existe que dans quelques dizaines d'années le problème de manque d'eau ne serait plus le problème de seule Afrique, mais de toute notre planète. Alors il faut penser comment économiser l'eau au quotidien à partir de maintenant.

Cette phrase commence avec une hypothèse en position de sujet, ce qui constitue une mise en valeur explicite de la proposition qui suit, contrairement au simple usage de si.

A7 S2 - La première partie, c'est donc trois heures qui passe comme une seul avec une mise en scène excellente, avec une composition de plan exemplaire.

La préposition avec équivaut à la conjonction de coordination et.

A6 S2 - Je ne peux pas dire que c'est sa meilleure œuvre, mais le livre m'a parut intéressant et passionnant ce qu'est démontré par le fait que je l'ai fini en cinq jours.

Ce qu'est (= ce qui est) comporte un ce anaphorique qui reprend la proposition précédente, à laquelle il introduit une justification explicitée par démontré.

A8 S2 - D'ailleurs l'eau qui existe est souvent inapte à boire, et les peuples locaux vu leur ignorance ne savent pas la purifier. D'où de nombreuses infections intestinales et des épidémies à rechute.

D'où est aussi une suppléance par la voie anaphorique exprimant la conséquence. A7 S1 - Nous entendons toujours des reproches et des conseils. Pour éviter cela j'essais de me séparer du socium pour un certain temps, de rester seule avec mes pensées sans écouter d'autres personnes. Comme ça, je suis certaine que c'est mon propre choix.

Comme ça, où ça est un pronom anaphorique, exprime une conclusion. 


\subsubsection{Conclusion sur la liaison mixte}

29 La plupart des cas de suppléance relevés mettent en jeu des processus anaphoriques. Ainsi, connexion et anaphores sont associées pour assurer la cohésion des textes produits. Hiérarchiquement, le lien de continuité thématique assuré par l'anaphore semble plus fort que la relation marquée par les connecteurs, qui peut toujours être identifiée par inférence au cours de la lecture du texte.

\subsection{Explications des pratiques de liaison des propositions et des phrases, connecteurs et autres moyens}

30 Ces pratiques peuvent être dues à la langue maternelle qui se différencie de la langue étrangère et influe d'une certaine manière sur le traitement des liaisons. L'absence de connecteurs là où ils seraient nécessaires en français et le choix d'autres moyens pour marquer les liaisons peuvent s'expliquer par les différences entre les deux langues. Ainsi, dans la phrase complexe, l'azerbaïdjanais peut se passer de conjonctions.

1. 0 , gələnə biz bütün işlərimizi bitirərik. (Lui arriver, nous finirons toutes nos affaires) = «On finira toutes nos affaires avant qu'il arrive.» Dans cette phrase on n'utilise pas de conjonction, le «avant que» est utilisé dans le verbe même «gələnə». Donc, grammaticalement, on ne voit aucune conjonction dans la phrase.

2. Mən bunu edirəm sən pis vəziyyətdə qalmayasan. (Je le fais tu ne sois pas embêté) = « Je le fais pour que tu ne sois pas embêté." Là aussi, on ne voit pas de conjonction dans la phrase azerbaidjanaise, le « pour que » est exprimé par le verbe « ne sois pas » utilisé au subjonctif en azerbaidjanais aussi.

31 Cette absence de connecteurs est aussi due à des connaissances insuffisantes du français, en raison de la priorité accordée traditionnellement dans l'enseignement du FLE à la grammaire de phrase et de la place restreinte de la grammaire de texte, indispensable pourtant à la production d'écrits avec l'emploi, entre autres, de connecteurs.

Les conjonctions de coordination et de subordination sont étudiées en détails à l'intérieur de la phrase complexe, et l'enseignement des adverbes de liaison tout comme celui des groupes prépositionnels reste fragmentaire dans les méthodes de français langue étrangère comme Alter Ego+, Taxi, Edito, etc., et aussi dans les livres de grammaire comme Grammaire progressive du français, Grammaire des premiers temps. Une bonne partie des connecteurs qui servent à baliser le texte ne fait jamais l'objet d'un enseignement grammatical explicite.

Les points de grammaire française les plus fréquemment abordés de façon explicite

dans les matériels d'apprentissage sont des points de morphologie (articles, pronoms, formes verbales) plus que des points de syntaxe. (Vigner, 2004, p. 85)

Tous ces ouvrages se limitent à la grammaire de phrase, alors que le travail sur les connecteurs demande de se placer dans la grammaire de texte. Ainsi, les apprenants connaissent le sens mais pas l'usage de ces connecteurs et leurs fonctions dans le texte. D'où cette hésitation à « prendre le risque » d'en utiliser plus dans les textes produits. 


\section{Pistes pédagogiques et propositions d'activités}

Compte tenu de nos observations, comment aider les apprenants à utiliser les connecteurs logiques, cet outil linguistique si important dans la production écrite?

De manière générale (voir Bartning, 1997 et Vigner, 2004), il convient de travailler avec les apprenants sur la grammaire de texte et l'analyse du discours, en ne se limitant pas, comme la plupart des grammaires et des méthodes de FLE, à la grammaire de phrase. On insistera, comme le préconise le CECRL dans la compétence discursive, sur « la capacité de gérer et de structurer le discours » (p. 96), notamment « en termes de cohérence et de cohésion » (p. 98) :

- relier une série d'éléments courts, simples et distincts en un discours qui s'enchaîne (niveau B1) ;

- utiliser avec efficacité une grande variété de mots de liaison pour marquer clairement les relations entre les idées (niveau B1) ;

- utiliser un nombre limité d'articulateurs pour relier ses énoncés bien qu'il puisse y avoir quelques « sauts » dans une longue intervention (niveau B2) ;

- produire un texte clair, fluide et bien structuré, démontrant un usage contrôlé de moyens linguistiques de structuration et d'articulation (niveau C1).

Un travail minutieux s'impose sur différents types de production écrite au niveau universitaire. On pourrait défendre une progression en spirale qui permettrait d'habituer les apprenants à avoir conscience des usages variés des connecteurs.

Pour ce faire, une série d'activités peut être proposée (Beacco, 2010). Vu la complexité du sujet et les lacunes des apprenants concernant l'utilisation des connecteurs dans les écrits, nous proposerions des activités d'ordre implicite ainsi qu'explicite suivant l'axe « lire et écrire des textes subjectifs (des essais subjectifs) ».

\section{Activités explicites :}

- Repérage. Travailler sur les textes (déjà construits) en demandant aux apprenants d'identifier les connecteurs. Ce repérage démontre bien le fonctionnement et l'objectif des connecteurs. La focalisation sur le texte et non seulement sur la phrase permet aux apprenants de travailler l'organisation textuelle en réception et en production.

- Substitution. Proposer des exercices sur les synonymes des connecteurs peut également être une bonne manière de familiarisation avec les connecteurs. Par exemple, demander de trouver les synonymes de pourtant invite l'apprenant à trouver les synonymes dans le large champ des connecteurs. Ainsi ajoute-il dans sa base en même temps que le connecteur pourtant, les connecteurs néanmoins, cependant, toutefois.

- Adjonction. Les activités structurales telles que « relier les phrases par un connecteur » sont de bons exercices d'entrainement. Deux versions sont possibles dans ce genre d'exercices. On peut présenter une liste des connecteurs possibles, ou bien laisser l'apprenant trouver tout seul le connecteur approprié. Dans les deux cas, l'apprenant sera face à un choix plus ou moins difficile.

- Récriture. Améliorer un texte. Retravailler sur un texte produit permettrait à l'apprenant de voir et analyser ses erreurs.

\section{Activités implicites :}

- L'exercice de mise en ordre des phrases ou des paragraphes dans un texte. L'apprenant travaille ainsi non seulement sur un sujet grammatical, mais aura aussi la possibilité d'établir les différentes étapes du raisonnement. 
- Demander aux apprenants de produire un texte serait une optimisation de l'emploi des connecteurs dans une production authentique.

\section{En guise de conclusion}

Nous avons voulu comprendre comment des apprenants de français langue étrangère en Azerbaïdjan utilisent les connecteurs logiques dans la production écrite. Nous avons essayé, à travers cet article, de déceler des stratégies d'écriture autour des connecteurs. Ces stratégies variées sont liées à la maitrise globale de l'expression écrite. La révélation de certains manques a permis de dégager des pistes pédagogiques qui aideraient les apprenants à employer les connecteurs d'une manière plus efficiente et les enseignants à non seulement évaluer les textes avec plus de précision, mais aussi à optimiser l'apprentissage des connecteurs. La grammaire de texte est indispensable aux étudiants avancés pour maitriser la production écrite.

Pour étendre les observations du traitement des liaisons dans un texte et notamment de l'emploi des connecteurs, il serait bon de confronter les productions écrites d'apprenants de FLE de différents pays, ayant des langues maternelles de familles différentes, ainsi que les productions d'étudiants FLE et FLM pour voir si l'on peut dégager des invariants dans les stratégies d'écriture.

\section{BIBLIOGRAPHIE}

\section{Références}

ADAM, Jean-Michel. (2011). La linguistique textuelle. Paris : Armand Colin.

ADAM, Jean-Michel. (2017). Les textes : types et prototypes ( $4^{\mathrm{e}}$ éd.). Paris : Colin.

BARRÉ-De Miniac, Christine, Brissaud, Catherine \& RisPaIL, Marielle. (2004). La littéracie.

Conceptions théoriques et pratiques d'enseignement de la lecture-écriture. Paris : L'Harmattan.

BARTNING, Inge. 1997. L'apprenant dit avancé et son acquisition d'une langue étrangère.

Acquisition et interaction en langue étrangère, 9, 9-50.

BEACCO, Jean-Claude. (2010). La didactique de la grammaire dans l'enseignement du français et des

langues. Paris : Didier.

CORNAIRE, Claudette \& RAYMOND, Patricia Mary. (1999). La production écrite. Paris : CLE International.

Riegel, Martin, Pellat, Jean-Christophe \& Rioul, René. (2016). Grammaire méthodique du français. Paris : PUF.

RUQUET, Michel \& QUOY-BODIN, Jean-Luc. 1998. Comment dire ? Raisonner à la française. Paris : CLE International.

VIGNER, Gérard. (2004). La grammaire en FLE. Paris : Hachette. 


\section{Méthodes de français consultées}

Abou-SAmra, Myriam, Heu, Élodie, Perrard, Marion \& Pinson, Cécile. (2012). Édito. Paris : Didier.

ABRY, Dominique \& CHALARON, Marie-Laure. (2015). La grammaire des premiers temps B1-B2 (nouvelle édition). Grenoble : PUG.

Berthet, Anne, Hugot, Catherine, SAmpsonis, Beatrix \& WAEndendries, Monique. (2012). Alter Ego + (niveaux 2, 3, 4). Paris : Hachette.

MAIA, Grégoire. (1996). Grammaire progressive du français, niveau B. Paris : CLE International.

MÉNARD, Robert. (2006). Taxi. Paris : Hachette.

\section{NOTES}

1. Les exemples sont présentés comme dans l'original : aucune modification ni correction n'y ont été apportées.

\section{RÉSUMÉS}

Les évaluations des compétences des étudiants en français constituent des révélateurs de leurs difficultés, notamment dans leurs connaissances de la langue et dans les quatre compétences. Parmi celles-ci, la littéracie constitue un enjeu majeur et un objectif essentiel pour les enseignants de FLE. À travers cet article, on s'intéressera en particulier à l'utilisation des connecteurs par les étudiants azerbaïdjanais qui ont un niveau avancé. Les connecteurs, qui sont un outil indispensable pour organiser le texte et le discours, illustrent bien le lien entre la grammaire et la littéracie, entre la connaissance de la langue et les capacités de compréhension et d'expression écrites, de comprendre et de produire des textes de différents types. Nous essayons de voir comment les apprenants azerbaïdjanais emploient les connecteurs ou des moyens équivalents et nous proposons des pistes didactiques.

The evaluations of the skills of the learners in French constitute revelations of their difficulties, in particular in their knowledge of the language and in four skills. Among these, les literacy constitutes a major stake and an essential objective for the teachers of French as foreign language. Through this article, we shall be interested in particular in the use of connectors by the Azerbaijani leaners who have an advanced level. Connectors, which are an essential tool to organize the text and the speech, illustrate well the link between the grammar and the literacy, between the knowledge of the language and the capacities of understanding and written expression, to understand and to produce texts of various types. We try to see how the learners use connectors or equivalent means and we propose educational trails. 
INDEX

Keywords : French as a foreign language, written expression, text, connectors, coherence, cohesion

Mots-clés : français langue étrangère, expression écrite, texte, connecteurs, cohérence, cohésion

\section{AUTEUR}

\section{SABINA MAHMUDOVA}

Université des langues d'Azerbaïdjan, Bakou 